\section{PD-L1-Antikörper Durvalumab verlängert Überleben bei NSCLC im Stadium III}

Antonia SJ et al. Durvalumab after Chemoradiotherapy in Stage III Non-Small-Cell Lung Cancer. New Engl J Med 2017; 377: 1919 - 1929

Zum Diagnosezeitpunkt eines nicht kleinzelligen Bronchialkarzinoms (NSCLC) befinden sich ein Drittel der Betroffenen bereits in Stadium III (lokal fortgeschritten, nicht resektabel). Trotz platinbasierter Chemotherapie und Bestrahlung beträgt das progressionsfreie Überleben hier im Median nur 8 Monate. Effekt und Sicherheit des monoklonalen Antikörpers Durvalumab nach einer Radiochemotherapie beim NSCLC Stadium III prüften Antonia et al. in dieser Phase-3-Studie.

Durvalumab, ein selektiver, hochaffiner humaner monoklonaler IgG1-Antikörper richtet sich gegen den Programmeddeath-Liganden 1 (PD-L1). Der Antikörper verhindert die Bindung von PD-L1 an PD1 sowie an CD80 mit dem Effekt, dass T-Zellen die Tumorzellen erkennen und vernichten können. Durvalumab wurde kürzlich in den USA zur Therapie des fortgeschrittenen Urothelkarzinoms nach platinbasierter Chemotherapie zugelassen. Studien zufolge wird die PDL1-Expression in Tumorzellen möglicherweise infolge einer Radiochemotherapie hochreguliert: Dies ist der pathophysiologische Hintergrund für die Hypothese, dass der PD-L1-Blocker Durvalumab beim NSCLC nach Radiochemotherapie günstige Wirkungen zeigen könnte.
An dieser randomisierten, placebokontrollierten Studie nahmen 709 Patienten mit NSCLC im Stadium III teil, die weder an einer Immundefizienz noch einem chronischen Infekt litten und noch nie mit einem PD1- oder PD-L1-Antikörper behandelt worden waren. Alle wiesen nach mindestens 2 Zyklen einer platinbasierten Chemotherapie plus Radiotherapie keine Krankheitsprogression auf. In einem Verhältnis von 2:1 erhielten 473 von ihnen Durvalumab $(10 \mathrm{mg} / \mathrm{kg}$ Körpergewicht i. v.) und 236 Placebo. Die Injektionen erfolgten 1 bis 42 Tage nach Beendigung der Radiochemotherapie zweiwöchentlich über meist 12 Monate. Im Median erhielten die Patienten im Verlauf 20 Dosen des Verums bzw. 14 Dosen Placebo. Unter Durvalumab ergab sich in einer geplanten Zwischenanalyse ein progressionsfreies Überleben (primärer Endpunkt) von im Median 16,8 Monaten (95\% Konfidenzintervall 13,0-18,1) versus 5,6 Monate unter Placebo (95\% Cl 4,6-7,8). Damit ließ sich eine Hazard Ratio für Tod oder Krankheitsprogression von 0,52 (95\% Cl 0,42-0,65; $p<0,001)$ errechnen. Mindestens 12 Monate ohne Progression überlebten 55,9\% versus 35,3\% (Placebo) der Patienten; die entsprechenden Werte für 18 Monate lagen bei $44,2 \%$ versus $27,0 \%$ zugunsten des Verum. Die Patienten wurden stratifiziert in Bezug auf Geschlecht, Alter $(>\mid<$ 65 Jahre), Raucherstatus und andere demografische Parameter. Der Effekt von Durvalumab auf das progressionsfreie Überleben zeigte sich hier unabhängig vom PD-L1-Spiegel vor der Radiochemotherapie; auch Nie-Raucher profitierten von dem Antikörper. Ansprechrate und -dauer waren unter Durvalumab im Vergleich günstiger, Fernmetastasen traten später auf und insbesondere neue Hirnmetastasen in der Verumgruppe auch seltener als bei den anderen. In beiden Gruppen erlitten etwa gleich viele $\mathrm{Pa}$ tienten schwere unerwünschte Wirkungen $(29,9 \%$ unter Durvalumab versus 26,1\%), wobei die Pneumonie mit 4,4\% versus 3,8\% die häufigste Grad-3/4-Nebenwirkung darstellte. 15,4\% der Patienten setzten Durvalumab aufgrund unerwünschter Wirkungen ab; dies galt für 9,8\% der anderen Probanden.

\section{FAZIT}

In der von Astra-Zeneca finanzierten Studie ergab sich ein deutlich verlängertes progressionsfreies Überleben für NSCLC-Patienten im Stadium III durch Durvalumab nach Radiochemotherapie gegenüber Placebo. Der günstige Effekt trat hierbei unabhängig von der PD-L1-Expression auf den Tumorzellen auf. Auch für die Sicherheit des Antikörpers ergaben sich positive Daten. Wie Effekte der Radiochemotherapie mit denen der Immuntherapie wechselwirken, sollte noch genauer untersucht werden, so die Autoren.

Dr. med. Susanne Meinrenken, Bremen 